\title{
Reproductive factors influencing bone mineral density in postmenopausal women
}

\begin{abstract}
Objective: This study examined the relationship between reproductive characteristics and bone mineral density in postmenopausal women who had been referred to the menopause clinics of the National Population and Family Development Board and of the Hospital Kuala Lumpur from July 2011 to January 2012. Method: The participants of this study were 201 postmenopausal Malaysian women aged 45-71 years. Some socio-demographic, life style and reproductive factors were recorded. Calcaneal bone mineral density was measured by quantitative ultra-sonography. Correlations of reproductive factors with bone mineral density were assessed by Pearson's correlation test and multiple regression analysis. Results: Age at menopause was not significantly correlated with bone mineral density, while the years after menopause, age at the first menstrual period, number of pregnancies and total lactation periods were inversely correlated with it. Among reproductive factors, only the association between lactation duration and bone mineral density remained significant after adjusting for age, body mass index, activity, and calcium intake. Conclusion: The results indicated that except for prolonged total time of lactation, other reproductive factors were not significantly associated with bone mineral density in post-menopausal women.
\end{abstract}

Keyword: Bone mineral density; Osteoporosis; Reproductive factors; Postmenopausal 